\title{
Human capital values and returns: Bounds implied by earnings and asset returns data
}

\author{
Mark Huggett ${ }^{\text {a }}$, Greg Kaplan ${ }^{\text {,** }}$

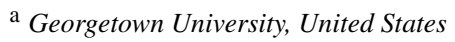 \\ ${ }^{\mathrm{b}}$ University of Pennsylvania, Department of Economics, 3718 Locust Walk, Philadelphia, PA, United States
}

Received 28 January 2010; final version received 20 July 2010; accepted 13 September 2010

Available online 17 November 2010

\begin{abstract}
We provide theory for calculating bounds on both the value of an individual's human capital and the return on an individual's human capital, given knowledge of the process governing earnings and financial asset returns. We calculate bounds using U.S. data on male earnings and financial asset returns. The large idiosyncratic component of earnings risk implies that bounds on values and returns are quite loose. However, when aggregate shocks are the only source of earnings risk, both bounds are tight.
\end{abstract}

(c) 2010 Elsevier Inc. All rights reserved.

JEL classification: J24; G12

Keywords: Value of human capital; Return on human capital; Asset pricing

\section{Introduction}

A long-standing problem is to provide an empirical description of the value of an individual's human capital and the associated return on an individual's human capital. The value of human capital is in theory simply discounted future earnings. Thus, it is key to determine how an individual's earnings and an individual's stochastic discount factor comove. The main difficulty is that discount factor properties can only be inferred indirectly through data on financial asset returns or individual choices.

\footnotetext{
We thank Adrien Verdelhan, Alisdair McKay, two anonymous referees and the associate editor for useful comments.

* Corresponding author. Fax: +1 2155732057.

E-mail addresses: mh5@georgetown.edu (M. Huggett), gkaplan@sas.upenn.edu (G. Kaplan).
} 
One strategy for making progress on this problem is to take a structural approach and make parametric assumptions about preferences, as well as assumptions on the exact structure of an individual's decision problem. These parameters can then be estimated, and the value and return to human capital can be characterized using the stochastic discount factor produced by a solution to an empirically-motivated specification of this decision problem.

In this paper we take a different approach. We explore what can be said about individual human capital values and returns without making parametric assumptions on preferences and without solving such a decision problem. However, we assume that one knows two important things:

(1) a statistical model for financial asset returns and an individual's earnings; and

(2) some key properties of an individual's stochastic discount factor.

We assume this discount factor is non-negative, satisfies an Euler equation for each financial asset and is no more variable than some specified upper bound. These assumptions will not allow one to precisely value an individual's future earnings unless future earnings can be replicated by trade in financial assets. Nevertheless, upper and lower bounds on the value of human capital can be determined by pricing the earnings component that can be replicated by trade in financial assets and then bounding the value of the residual component of earnings.

We view the two approaches as being complementary. If the bounds approach puts tight bounds on values and returns, then this tells one that all the extra assumptions and additional data used in the structural approach can only serve to slightly narrow the value and return to human capital beyond what can be determined from earnings and asset returns data. In contrast, if the bounds approach implies very loose bounds, then this tells one that the additional data and assumptions employed in the structural approach are critical for reaching conclusions about the return to human capital.

We highlight one area in which an empirical understanding of the value and return to human capital is relevant. To maintain a constant fraction of overall wealth in stock holdings, an individual's direct financial holdings of stock and bonds need to be selected with the value of human capital in mind. If human capital is like stock, then the fraction of financial wealth held in stock would need to increase over the lifetime. If human capital is like risk-free debt, then the opposite reasoning applies. To make progress on this argument and give practical advice, one needs to investigate this if condition empirically. To do so, it is important to adopt the human capital value and return notions used in this paper: values and returns based on an individual's stochastic discount factor.

There are three main contributions of the paper. First, we show that value bounds imply return bounds. Second, we illustrate how all the concepts work within a simple example. Third, we calculate value and return bounds using U.S. data.

Value and return bounds for U.S. data are determined in two steps. We start by providing an empirical description of the joint dynamics of male earnings and stock returns. Given such a statistical model, we then calculate value and return bounds using the restriction that the coefficient of variation of an individual's stochastic discount factor is no larger than a given multiple of the conditional Sharpe ratio. If the Euler equation restriction is to hold, then this coefficient of variation must, at a minimum, be at least as large as the Sharpe ratio. We find that value and return bounds are very loose even after imposing that the coefficient of variation is at most 1.1 times the conditional Sharpe ratio. Specifically, for this upper limit the expected lifetime return to human capital must lie between -10 and 17 percent per year. This is almost exclusively due to the 
large amount of idiosyncratic earnings variation that we estimate from U.S. data, consistent with findings from numerous previous empirical studies. We find that when all idiosyncratic risk is eliminated without eliminating aggregate sources of earnings risk, then value and return bounds are tight. The expected lifetime return to human capital is then between 0.25 and 2.5 percent per year, for a range of restrictions on the coefficient of variation of an individual's stochastic discount factor.

Three literatures are most closely related to the problem that we address. First, there is a literature on the value of human capital. This literature has almost exclusively focused on valuing highly aggregated measures of cash flows (e.g. economy-wide earnings or cohort earnings) rather than individual male earnings as examined in this paper. See Huggett and Kaplan [6] for a discussion of this literature. Second, the finance literature has put upper and lower value bounds on cash flows. Cochrane and Saa-Requejo [3] and Cochrane [2] develop theory, provide applications and review this literature. The bounds literature builds on the stochastic discount factor formulation of asset pricing problems developed by Hansen and Jagannathan [5] and others. The basic idea in the value bounds literature is to value the component of cash flows that can be replicated by trade in marketed assets and bound the value of the residual component. To the best of our knowledge, we are the first to apply these ideas to calculate value and return bounds on individual-level earnings. Third, the paper is related to the literature on incomplete markets and idiosyncratic earnings risk. Specifically, market incompleteness is what generates a gap between upper and lower value bounds and is hence key to our analysis.

\section{Framework}

\subsection{Basic concepts}

We assume that the asset pricing theorist knows the stochastic process governing earnings $e=$ $\left\{e_{j}\right\}_{j=1}^{J}$ and (gross) financial asset returns $R^{i}=\left\{R_{j}^{i}\right\}_{j=1}^{J}$ for $i \in I . J$ denotes the last period of the working lifetime, whereas $I$ denotes the set of assets that the agent observes and can trade. The agent values future earnings using a stochastic discount factor $m^{*}=\left\{m_{j, j+1}^{*}\right\}_{j=1}^{J}$. The discount factor between age $j$ and $k$ is the product of one-period discount factors $m_{j, k}^{*}=\prod_{i=j}^{k-1} m_{i, i+1}^{*}$. All random variables are defined on some underlying probability space $(\Omega, \mathcal{F}, P)$ and are assumed to be square integrable.

We now define the value of human capital and the return to human capital. The value of an agent's human capital $v_{j}\left(m^{*}\right)$ is simply the value of future earnings discounted using $m^{*}$. Expected values at age $j$ are calculated using the information $\sigma$-field $\mathcal{F}_{j}$ of the agent. The oneperiod discount factor $m_{j, j+1}^{*}$ is assumed to be $\mathcal{F}_{j+1}$ measurable. We assume that $\mathcal{F}_{j}$ is the $\sigma$-field generated by earnings and asset returns up to period $j$ (i.e. $\left(e_{k},\left(R_{k}^{i}\right)_{i \in I}\right)$ for $k \leqslant j$ ). ${ }^{1}$ The return to human capital $R_{j+1}^{h}\left(m^{*}\right)$ is defined as the random variable which equals the sum of the earnings and value in period $j+1$ divided by the period $j$ value. The lifetime return $R_{\text {life }}\left(m^{*}\right)$ is the geometric average of the period returns

\footnotetext{
1 The theory can be extended to cover the case where $\mathcal{F}_{j}$ is finer as would be the case when the agent also observes signals providing information on future earnings and returns beyond those implied by past earnings and returns.
} 


$$
\begin{aligned}
& v_{j}\left(m^{*}\right) \equiv E\left[\sum_{k=j+1}^{J} m_{j, k}^{*} e_{k} \mid \mathcal{F}_{j}\right] \\
& R_{j+1}^{h}\left(m^{*}\right) \equiv \frac{v_{j+1}\left(m^{*}\right)+e_{j+1}}{v_{j}\left(m^{*}\right)} \\
& R_{\text {life }}\left(m^{*}\right) \equiv\left[R_{2}^{h}\left(m^{*}\right) R_{3}^{h}\left(m^{*}\right) \cdots R_{J}^{h}\left(m^{*}\right)\right]^{1 /(J-1)} .
\end{aligned}
$$

Huggett and Kaplan [6] provide a justification for defining the value of human capital in this way. They argue that if an agent is given the opportunity to move away from holding all the shares in his/her cash flow stream $e_{j}$, then when the value of these shares is set at $v_{j}\left(m^{*}\right)$ the agent will optimally choose to always continue to hold all these shares. While the argument for this proposition relies on a concave utility function over consumption, it is not sensitive to the exact theoretical mechanism by which earnings are produced. If earnings are produced with goods inputs, then it is earnings net of the value of goods inputs that must be valued.

\subsection{Bounds}

We assume that the asset pricing theorist knows that the agent's discount factor lies in the set $M$. This set contains square integrable stochastic discount factors that satisfy three restrictions. First, each one-period discount factor is non-negative. Second, the Euler equation holds for each financial asset available to the agent. These first two restrictions would be implied, from a utility theory framework, by a strictly positive marginal utility of consumption and by agent optimization. Third, all the one-period discount factors have a second moment that is no larger than some upper bound denoted $b^{2}$. This last restriction is an auxiliary assumption rather than a restriction implied by some underlying decision problem

$$
\begin{aligned}
M \equiv & \left\{m: \text { (1) } m_{j, j+1} \geqslant 0, \text { (2) } E\left[m_{j, j+1} R_{j+1}^{i} \mid \mathcal{F}_{j}\right]=1, \forall i \in I,\right. \\
& \text { (3) } \left.E\left[m_{j, j+1}^{2} \mid \mathcal{F}_{j}\right] \leqslant b^{2}, \forall j\right\} .
\end{aligned}
$$

The upper and lower bounds on values and returns are simply the supremum and infimum of the possible values and returns over the set $M$. These are defined below. Since values and returns are random variables the bounds are also random variables

$$
\begin{aligned}
& v_{j}^{u} \equiv \sup _{m \in M} v_{j}(m) \quad \text { and } \quad v_{j}^{l} \equiv \inf _{m \in M} v_{j}(m) \\
& R_{\text {life }}^{u} \equiv \sup _{m \in M} R_{\text {life }}(m) \quad \text { and } \quad R_{\text {life }}^{l} \equiv \inf _{m \in M} R_{\text {life }}(m) .
\end{aligned}
$$

It is important to clarify when there will be a gap between the upper bound $v_{j}^{u}$ and the lower bound $v_{j}^{l}$. Any gap between the value bounds will then imply a gap between the return bounds. There can be a gap between the value bounds when it is not possible to replicate future earnings with a trading strategy in financial assets. Thus, market incompleteness is key. To see this in its simplest form, consider the value in the second-to-last period $v_{J-1}(m)$. Below we rewrite $v_{J-1}(m)$ using the fact that there is a unique decomposition $e_{J}=\operatorname{proj}_{J}\left(e_{J}\right)+\epsilon_{J}$ of earnings into the part $\operatorname{proj}_{J}\left(e_{J}\right)$ in the space spanned by financial asset returns and the orthogonal part. The component in the space spanned by asset returns has exactly the same value for any $m \in M$. This fact is implied by the Euler equation and is expressed in the second equation below using $\operatorname{proj}_{J}\left(e_{J}\right)=\sum_{i \in I} \alpha_{J-1}^{i} R_{J}^{i}$ for some weights $\alpha_{J-1}^{i}$, 


$$
\begin{aligned}
& \begin{aligned}
v_{J-1}(m) & =E\left[m_{J-1, J}\left(\operatorname{proj}_{J}\left(e_{J}\right)+\epsilon_{J}\right) \mid \mathcal{F}_{J-1}\right] \\
& =E\left[m_{J-1, J} \operatorname{proj}_{J}\left(e_{J}\right) \mid \mathcal{F}_{J-1}\right]+E\left[m_{J-1, J} \epsilon_{J} \mid \mathcal{F}_{J-1}\right]
\end{aligned} \\
& E\left[m_{J-1, J} \operatorname{proj}_{J}\left(e_{J}\right) \mid \mathcal{F}_{J-1}\right]=E\left[m_{J-1, J} \sum_{i \in I} \alpha_{J-1}^{i} R_{J}^{i} \mid \mathcal{F}_{J-1}\right]=\sum_{i \in I} \alpha_{J-1}^{i}, \quad \forall m \in M .
\end{aligned}
$$

This decomposition makes it clear that any gap between the upper and lower value bounds comes about when the value of the orthogonal component of earnings differs across $m \in M$. This happens when the orthogonal component $\epsilon_{J}$ is non-zero and when there are two discount factors $m^{1}, m^{2} \in M$ that covary differently with this component of earnings. The first criteria is an observable as the asset pricing theorist is assumed to know the statistical process governing earnings and asset returns. In practice, empirical methods can provide evidence on the size and nature of the orthogonal component of earnings. The second criteria occurs when the volatility bound (restriction (3)) is large enough so that $m$ is sufficiently variable to satisfy the Euler equations (restriction (2)) and there is some additional scope for $m$ to vary with the orthogonal component of earnings.

\subsection{Relationship between value bounds and return bounds}

Next, we show that bounds on lifetime returns are implied by bounds on the value of human capital. To see why this holds, simply restate lifetime returns as indicated below. It is then clear that, for any draw $\omega \in \Omega$ of shocks, lower values for human capital imply higher values for lifetime returns. Thus, an upper bound on lifetime returns is obtained by inserting the lower bound on values $v_{j}^{l}$ at each age into the right-hand side of this equation. Using the same logic, a lower bound on lifetime returns is determined by the upper bound on values $v_{j}^{u}$. Theorem 1 argues that the least upper bound and the greatest lower bound on lifetime returns are determined by the value bounds

$$
\begin{aligned}
R_{\text {life }}(m) & \equiv\left[\left(\frac{v_{2}(m)+e_{2}}{v_{1}(m)}\right) \cdots\left(\frac{v_{J}(m)+e_{J}}{v_{J-1}(m)}\right)\right]^{1 /(J-1)} \\
& =\left[\frac{e_{J}}{v_{1}(m)} \prod_{j=2}^{J-1}\left(1+\frac{e_{j}}{v_{j}(m)}\right)\right]^{1 /(J-1)}
\end{aligned}
$$

Theorem 1. Let $\left(m^{*}, e,\left[R^{i}: i \in I\right]\right)$ denote the agent's stochastic discount factor, earnings and asset returns. If earnings and asset returns are strictly positive and the set of possible stochastic discount factors $M$ is non-empty, then

(i) $R_{\text {life }}^{l}=\left[\frac{e_{J}}{v_{1}^{u}} \prod_{j=2}^{J-1}\left(1+\frac{e_{j}}{v_{j}^{u}}\right)\right]^{1 /(J-1)}$ and $R_{\text {life }}^{u}=\left[\frac{e_{J}}{v_{1}^{l}} \prod_{j=2}^{J-1}\left(1+\frac{e_{j}}{v_{j}^{l}}\right)\right]^{1 /(J-1)}$.

(ii) $R_{\text {life }}\left(m^{*}\right) \in\left[R_{\text {life }}^{l}, R_{\text {life }}^{u}\right]$ provided that the volatility bound is above the second moment of the agent's stochastic discount factor (i.e. $\left.b^{2} \geqslant E\left[\left(m_{j, j+1}^{*}\right)^{2} \mid \mathcal{F}_{j}\right], \forall j\right)$.

Proof. See Appendix D. 


\section{A simple example}

In this section we provide a simple example to illustrate how all the concepts work. We start by describing an environment with a single risk-free asset and permanent idiosyncratic earnings shocks. This model is a finite lifetime version of the problem analyzed by Constantinides and Duffie [4]. This model is useful because it allows us to express both the value and the return to human capital in closed form. We then calculate bounds, assuming that only the earnings process and the interest rate are known, and not the full structure of the model.

\subsection{The model}

Consider the decision problem of an agent who faces an exogenous earnings process and can borrow and lend at a risk-free rate $r$. Earnings are subject to permanent shocks arriving each model period. The agent maximizes expected utility over the lifetime.

Utility:

$$
E\left[\sum_{j=1}^{J} \beta^{j-1} u\left(c_{j}\right) \mid \mathcal{F}_{1}\right], \quad \text { where } u(c)= \begin{cases}\frac{c^{1-\rho}}{(1-\rho)} & \rho>0, \rho \neq 1 \\ \log (c) & \rho=1 .\end{cases}
$$

Earnings: $e_{j}=\prod_{k=1}^{j} z_{k}$, where $\ln z_{k} \sim N\left(\mu, \sigma^{2}\right)$ is i.i.d.

Information: $\mathcal{F}_{j}$ is the $\sigma$-field generated by $\left(e_{1}, \ldots, e_{j}\right)$.

Risk-free return: $R=(1+r)>0$.

Decision problem:

$$
\begin{aligned}
& \max E\left[\sum_{j=1}^{J} \beta^{j-1} u\left(c_{j}\right) \mid \mathcal{F}_{1}\right] \quad \text { subject to } \\
& \begin{array}{l}
\text { (1) } c_{j}+a_{j+1} \leqslant a_{j}(1+r)+e_{j}, \quad \text { (2) } \quad c_{j} \geqslant 0, a_{J+1} \geqslant 0 .
\end{array}
\end{aligned}
$$

When the interest rate satisfies $1+r=\frac{1}{\beta} \exp \left(\rho \mu-\frac{\rho^{2} \sigma^{2}}{2}\right)$ and initial assets are zero, then the solution to the agent's decision problem is to set consumption equal to earnings each period. The plausibility of this assertion can be seen by noting that the agent's Euler equation holds under these conditions. The agent's stochastic discount factor is then $m_{j, j+1}^{*}=\beta \frac{u^{\prime}\left(c_{j+1}\right)}{u^{\prime}\left(c_{j}\right)}=\beta \frac{u^{\prime}\left(e_{j+1}\right)}{u^{\prime}\left(e_{j}\right)}$. The agent's value of human capital $v_{j}\left(m^{*}\right)$ is then proportional to earnings each period. This implies that the agent's realized return to human capital $R_{j+1}^{h}\left(m^{*}\right)$ is proportional to the permanent earnings shock $z_{j+1}$. Lifetime returns are then easy to determine as they are the geometric average of realized period returns and period returns are i.i.d. across periods. These results are summarized below

$$
\begin{aligned}
& v_{j}\left(m^{*}\right)=E\left[\sum_{k=j+1}^{J} m_{j, k}^{*} e_{k} \mid \mathcal{F}_{j}\right]=f_{j} e_{j} \\
& f_{j}=\sum_{k=j+1}^{J} \beta^{k-j} \exp \left((k-j)\left[(-\rho+1) \mu+(-\rho+1)^{2} \frac{\sigma^{2}}{2}\right]\right)
\end{aligned}
$$




$$
\begin{aligned}
& R_{j+1}^{h}\left(m^{*}\right)=\frac{e_{j+1}+f_{j+1} e_{j+1}}{f_{j} e_{j}}=\left(\frac{1+f_{j+1}}{f_{j}}\right) z_{j+1} \\
& E\left[R_{\text {life }}\left(m^{*}\right) \mid \mathcal{F}_{1}\right]=\frac{1}{\beta} \exp \left(\mu \rho+\frac{\sigma^{2}}{2}\left(\frac{1}{J-1}-(1-\rho)^{2}\right)\right) .
\end{aligned}
$$

\subsection{Bounds on values and returns}

Next we consider an asset pricing theorist who has knowledge of the stochastic process for earnings and the interest rate on the single risk-free asset. We now determine what could be said about the value and return to the agent's human capital, given only this knowledge, and no other details of the decision-making environment.

We start by noting that upper and lower value bounds satisfy a Bellman equation. We focus on the Bellman equation for the upper bound since the lower bound can be handled by replacing the supremum with the infimum operation. The state variable in Bellman's equation is current earnings $e_{j}$ as earnings are Markovian

$$
\begin{aligned}
& v_{j}^{u}\left(e_{j}\right)=\sup _{m_{j, j+1}} E\left[m_{j, j+1}\left(v_{j+1}^{u}\left(e_{j+1}\right)+e_{j+1}\right) \mid e_{j}\right] \text { subject to } \\
& \begin{array}{ll}
\text { (1) } m_{j, j+1} \geqslant 0, & \text { (2) } E\left[m_{j, j+1}(1+r) \mid e_{j}\right]=1 \quad \text { and } \quad \text { (3) } E\left[m_{j, j+1}^{2} \mid e_{j}\right] \leqslant b^{2} .
\end{array}
\end{aligned}
$$

To solve Bellman's equation, two facts are useful. First, bounds are linear in current earnings: $v_{j}^{u}(e)=\gamma_{j}^{u} e$. This follows from backwards recursion using the fact that shocks are multiplicative $e_{j+1}=e_{j} z_{j+1}$ and $z_{j+1}$ is independent of $e_{j}$. Second, a simple recursion governs the coefficients: $\gamma_{j}^{u}=\left(\gamma_{j+1}^{u}+1\right) \gamma_{J-1}^{u}$, where $\gamma_{J}=0$. This implies that $\gamma_{j}^{u}=\sum_{k=j}^{J-1}\left(\gamma_{J-1}^{u}\right)^{J-k}$. Thus, calculating all the upper bounds boils down to finding $\gamma_{J-1}^{u}=$ $v_{J-1}^{u}(1)=\sup _{\{m:(1)-(3) h o l d\}} E\left[m_{J-1, J} z\right]$.

Bounds on returns are now easy to state for this model. Theorem 1 tells one that lower bounds on the realized lifetime returns are determined by upper bounds on the value of human capital. Furthermore, value bounds are proportional to realized earnings. These statements are summarized in the first equation below. The second and third equation below state bounds on mean lifetime returns based on the bound for realized returns. Theorem 1 implies that $E\left[R_{\text {life }}^{l} \mid \mathcal{F}_{1}\right] \leqslant E\left[R_{\text {life }}\left(m^{*}\right) \mid \mathcal{F}_{1}\right] \leqslant E\left[R_{\text {life }}^{u} \mid \mathcal{F}_{1}\right]$, provided that the exogenously conjectured volatility bound $b^{2}$ is at least as large as the second moment of the agent's stochastic discount factor. Shortly, we will use these equations to calculate bounds, given a computational method for approximating $\left(\gamma_{J-1}^{u}, \gamma_{J-1}^{l}\right)$,

$$
\begin{aligned}
& R_{\text {life }}^{l}=\left[\left(\frac{e_{2}+\gamma_{2}^{u} e_{2}}{\gamma_{1}^{u} e_{1}}\right) \cdots\left(\frac{e_{J-1}+\gamma_{J-1}^{u} e_{J-1}}{\gamma_{J-2}^{u} e_{J-2}}\right)\left(\frac{e_{J}}{\gamma_{J-1}^{u} e_{J-1}}\right)\right]^{1 /(J-1)} \\
& \quad=\frac{1}{\gamma_{J-1}^{u}}\left[\frac{e_{J}}{e_{1}}\right]^{1 /(J-1)} \\
& E\left[R_{\text {life }}^{l} \mid \mathcal{F}_{1}\right]=\frac{1}{\gamma_{J-1}^{u}} \exp \left(\mu+\frac{\sigma^{2}}{2(J-1)}\right) \\
& E\left[R_{\text {life }}^{u} \mid \mathcal{F}_{1}\right]=\frac{1}{\gamma_{J-1}^{l}} \exp \left(\mu+\frac{\sigma^{2}}{2(J-1)}\right) .
\end{aligned}
$$




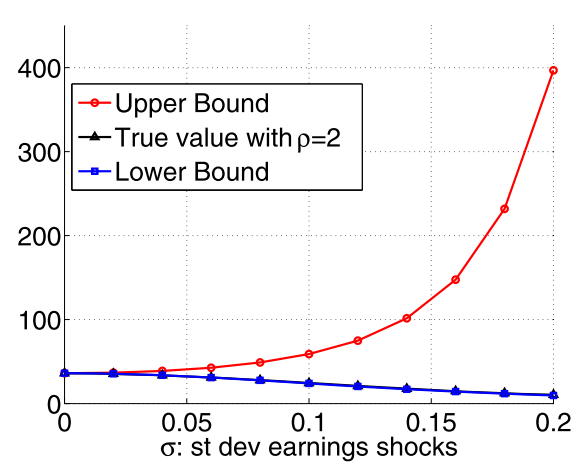

(a) Value Bounds

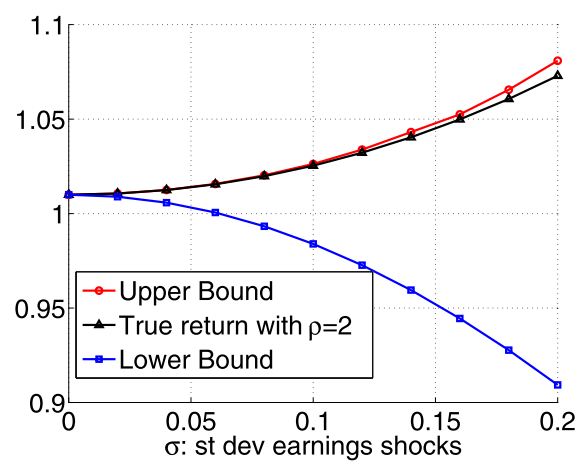

(b) Returns Bounds

Fig. 1. Bounds on values and return for model in Section 3, by standard deviation of earnings shocks $(\sigma)$.

\subsection{Quantitative properties of values and returns}

We analyze a quantitative version of this model to illustrate how all these ideas work together. The interest rate is set to $r=0.01$ and the agent's lifetime is set to $J=46$ model periods. Earnings parameters are set so that the mean earnings profile equals 1 each model period: $\mu=$ $-\sigma^{2} / 2$. We allow a one standard deviation shock to earnings to vary from zero to a twenty percent shock. These assumptions are sufficient to calculate bounds on values and returns. To calculate the true value and return to human capital, in addition it is necessary to specify the preference parameters, and to ensure that the interest rate assumption used in the construction of the solution to the decision problem holds. To achieve this, we set an agent's coefficient of relative risk aversion to $\rho=2$ and set the discount factor $\beta=\frac{\exp \left(\rho \mu-\frac{\rho^{2} \sigma^{2}}{2}\right)}{1+r}$.

Fig. 1(a) displays upper and lower value bounds for different magnitudes of the standard deviation $\sigma$ of log earnings shocks. ${ }^{2}$ These value bounds are based on an exogenously specified volatility bound which we set equal to the true second moment of the agent's stochastic discount factor (i.e. $b^{2} \equiv E\left[m_{j, j+1}^{* 2} \mid \mathcal{F}_{j}\right]$ ) for each value of $\sigma$. Fig. 1(a) shows that the bounds on values fan out as earnings risk increases. The true value of human capital $v_{1}\left(m^{*}\right)$ is also displayed in Fig. 1(a). The true value is difficult to see in this figure as it is always very close to the computed lower bound. ${ }^{3}$

Fig. 1(a) shows that the value of an agent's human capital falls as the shock variance increases. To help understand this result, below we decompose the value into two components: the value of the mean earnings profile and the value of the residual (orthogonal) component of earnings $\epsilon_{k} \equiv e_{k}-E\left[e_{k} \mid \mathcal{F}_{1}\right]$. These can be viewed as the value of the earnings component spanned by asset returns and the value of the orthogonal component. The first component is exactly the same for each model because both the mean earnings profile and the mean of the agent's stochastic discount factor are the same. Furthermore, the first component can be precisely valued without

\footnotetext{
2 The bounds are calculated using the theory from the previous subsection and using computational methods to calculate $\left(\gamma_{J-1}^{u}, \gamma_{J-1}^{l}\right)$. To compute these two quantities, we discretize the shock process and numerically solve the optimization problem. All programs used to compute results can be found at the journal's website.

3 This is not too surprising. The agent's true stochastic discount factor comoves negatively with earnings. The stochastic discount factor that achieves the lower bound on the value of human capital will also comove negatively with earnings and will by construction have the same first two moments as the agent's true stochastic discount factor.
} 
knowledge of the precise nature of the agent's stochastic discount factor $m^{*}$. Thus, the value of human capital is lower with risk than in the no earnings risk case because the value of the orthogonal component of earnings is negative with risk. This occurs because an agent's stochastic discount factor covaries negatively with the orthogonal component of earnings

$$
v_{1}\left(m^{*}\right)=E\left[\sum_{k=2}^{J} m_{1, k}^{*} e_{k} \mid \mathcal{F}_{1}\right]=\sum_{k=2}^{J} E\left[m_{1, k}^{*} E\left[e_{k} \mid \mathcal{F}_{1}\right] \mid \mathcal{F}_{1}\right]+\sum_{k=2}^{J} E\left[m_{1, k}^{*} \epsilon_{k} \mid \mathcal{F}_{1}\right] .
$$

Fig. 1(b) displays the mean lifetime return on human capital and the upper and lower bound on expected lifetime returns. These are calculated based upon the formulas derived in Section 3.2. Fig. 1(b) shows that the true mean lifetime return increases as risk increases and that the upper bound on lifetime returns closely approximates the agent's true mean return.

The cone-shaped areas in Fig. 1 can be interpreted as follows. An empirical researcher could impose parametric assumptions on the utility function and make specific assumptions on the nature of an agent's budget constraint. Given a procedure for selecting these parameters, the value and return to human capital could be calculated using the stochastic discount factor resulting from a solution to the agent's decision problem. Applying such a procedure for each value of the earnings risk $\sigma$, would produce a map or correspondence living within the cone-shaped areas of Fig. 1. This holds regardless of the parametric class of utility functions that the researcher specifies, provided that the Euler equation restriction holds. However, it is key that the second moment of the agent's stochastic discount factor inferred from such a parametric procedure is not above the value employed in calculating the bounds.

\subsection{Additional restrictions on $M$}

Within the simple example analyzed, it may seem unreasonable that the class $M$ of stochastic discount factors allows discount factors to covary positively with the orthogonal component of earnings. However, this is precisely what happens at the upper bound on values and the lower bound on returns. Thus, one can ask whether or not theory puts restrictions on $M$ beyond those employed so far. For example, is the covariation always non-positive with the orthogonal component of earnings?

Claim 1 presents a simple result on this question. It proves that within a two-period model with exogenous earnings this covariation is always non-positive. In this claim $E_{2}$ denotes the expectations operator conditioned on period 2 asset returns.

\section{Problem P1.}

$$
\begin{aligned}
& \max E_{1}\left[U\left(c_{1}, c_{2}\right)\right] \\
& \text { subject to } \\
& c_{1}+a_{2} \leqslant a_{1} \sum_{i \in I} \alpha_{1}^{i} R_{1}^{i}+e_{1} ; \quad c_{2} \leqslant a_{2} \sum_{i \in I} \alpha_{2}^{i} R_{2}^{i}+e_{2} .
\end{aligned}
$$

Claim 1. Assume $U\left(c_{1}, c_{2}\right)$ is strictly increasing, strictly concave and differentiable. At a solution to Problem $\mathrm{P} 1$, where $m^{*}=\frac{U_{2}\left(c_{1}, c_{2}\right)}{E_{1}\left[U_{1}\left(c_{1}, c_{2}\right)\right]}$ and $\epsilon_{2} \equiv e_{2}-E_{2}\left[e_{2}\right]$, we have that

(1) $E_{1}\left[m^{*} \epsilon_{2}\right] \leqslant 0$; and

(2) $v_{1}\left(m^{*}\right) \equiv E_{1}\left[m^{*} e_{2}\right] \leqslant E_{1}\left[m^{*} E_{2}\left[e_{2}\right]\right]$. 
Proof. (1) $E_{1}\left[m^{*} \epsilon_{2}\right]=E_{1}\left[E_{2}\left[m^{*} \epsilon_{2}\right]\right] \leqslant E_{1}\left[E_{2}\left[m^{*}\right] E_{2}\left[\epsilon_{2}\right]\right]=0$. The leftmost equality holds by the law of iterated expectations. The inequality holds as the conditional covariance between $m^{*}$ and $\epsilon_{2}$ is non-positive. This holds as, conditioned on second period asset returns, $m^{*}$ decreases as $\epsilon_{2}$ increases by concavity. The rightmost equality holds as $E_{2}\left[\epsilon_{2}\right]=0$. (2) This is implied by (1).

Claim 1 implies that in the simple example from Section 3.1, with only two model periods $(J=2)$ and with a flat mean earnings profile, the upper bound on values and the lower bound on returns are now tighter: $v_{1}\left(m^{*}\right)=E_{1}\left[m^{*} e_{2}\right] \leqslant e_{1} / R$ and $E_{1}\left[R_{2}^{h}\right]=E_{1}\left[\frac{e_{2}}{v_{1}\left(m^{*}\right)}\right] \geqslant$ $E_{1}\left[\frac{e_{2}}{e_{1} / R}\right]=R$.

Extending this simple result beyond two periods is not straightforward for general earnings and returns structures. It would be sufficient to show that future consumption increases with a positive innovation to earnings, conditioned on returns. Establishing this is not so simple as future consumption now depends on an endogenous future savings decision.

We show that a multi-period analog to Claim 1 holds for the purely temporary component of earnings variation within models where earnings are exogenous and have a Markovian shock structure. Claim 2 presents this result when earnings $e_{j}=\bar{e}_{j} v_{j}$ are the product of an independent shock $v_{j}$ and a Markovian component $\bar{e}_{j}$. The earnings residual $\epsilon_{j}$ is then $\epsilon_{j} \equiv e_{j}-\hat{E}_{j}\left[e_{j}\right]$, where the expectations operator is conditioned on all period $j$ earnings and return information except the realization of the independent temporary shock $v_{j}$. The next section presents an empirical earnings and return model of this type.

\section{Problem P2.}

$\max E_{1}\left[\sum_{j=1}^{J} \beta^{j-1} u\left(c_{j}\right)\right]$

subject to

$c_{j}+a_{j+1} \leqslant a_{j} \sum_{i \in I} \alpha_{j}^{i} R_{j}^{i}+e_{j} ; \quad a_{J+1} \geqslant 0$.

Claim 2. Assume $u(c)$ is strictly increasing, strictly concave and differentiable and that earnings have an independent shock component which is multiplicative. At an interior solution to Problem $\mathrm{P} 2$, where $m_{j, j+1}^{*}=\frac{\beta u^{\prime}\left(c_{j+1}\right)}{u^{\prime}\left(c_{j}\right)}$, we have $E_{j}\left[m_{j, j+1}^{*} \epsilon_{j+1}\right] \leqslant 0, \forall j$.

Proof. Note that

$$
E_{j}\left[m_{j, j+1}^{*} \epsilon_{j+1}\right]=E_{j}\left[\hat{E}_{j+1}\left[m_{j, j+1}^{*} \epsilon_{j+1}\right]\right] \leqslant E_{j}\left[\hat{E}_{j+1}\left[m_{j, j+1}^{*}\right] \hat{E}_{j+1}\left[\epsilon_{j+1}\right]\right]=0 .
$$

The leftmost equality holds by the law of iterated expectations. The inequality holds provided the conditional covariance is non-positive. The rightmost equality holds as $\hat{E}_{j+1}\left[\epsilon_{j+1}\right]=0$.

It remains to show that the conditional covariance is non-positive. It is sufficient to show that $c_{j+1}$ is increasing in $v_{j+1}$, other things equal, as then $\epsilon_{j+1}$ increases and $m_{j, j+1}$ decreases as $v_{j+1}$ increases. Given the Markovian structure, we can pose Problem P2 recursively and view consumption $c_{j+1}\left(w_{j+1}, z_{j+1}, v_{j+1}\right)$ as a function of a vector of state variables, where $z_{j+1}$ summarizes the information in asset returns and all components of earnings other than the temporary shock $v_{j+1}$ and $w_{j+1}=a_{j+1} \sum_{i \in I} \alpha_{j+1}^{i} R_{j+1}^{i}$ summarizes financial wealth 


$$
\begin{aligned}
& v_{j}\left(w_{j}, z_{j}, v_{j}\right)=\max u\left(c_{j}\right)+\beta E_{j}\left[v_{j+1}\left(w_{j+1}, z_{j+1}, v_{j+1}\right)\right] \\
& c_{j}+a_{j+1} \leqslant w_{j}+e_{j} .
\end{aligned}
$$

A standard result for this type of problem is that at an interior solution the value function is increasing, concave and differentiable in $w$ and $d \frac{v_{j}\left(w_{j}, z_{j}, v_{j}\right)}{d w_{j}}=u^{\prime}\left(c_{j}\left(w_{j}, z_{j}, v_{j}\right)\right)$. These properties are established by backwards induction using the corresponding properties of $u$. Concavity of the value function then implies that $c_{j}$ must increase in $w_{j}$, other things equal. By independence, an increase in $v_{j}$ is equivalent to an appropriate increase in financial wealth. Thus, $c_{j}$ also must increase in $v_{j}$, other things equal.

\section{Earnings and asset returns: empirics}

We now turn to applying these ideas to calculate bounds on human capital values and returns using U.S. data. To operationalize the concepts introduced in Section 2 it is necessary to have knowledge of (1) the set of assets that are actively traded by households and (2) the joint stochastic structure of earnings and asset returns. Regarding the set of actively traded financial assets, we focus our analysis on the case of two assets: one risky (equities) and one riskless (bonds). We review evidence on how widely these two assets classes are held by U.S. households.

Campbell [1] analyzes participation rates of U.S. households in different asset classes using the 2001 Survey of Consumer Finances. He finds that safe assets (e.g. checking, saving and money market accounts) and vehicles are very widely held across households. These two asset classes are held by at least 80 percent of households within any percentile of the total asset holding distribution, except for households in the bottom 10 percent of the asset holding distribution. Thus, assuming that the Euler equation holds for a low-risk asset is well motivated. The evidence for holding equity is more mixed. Within the top 25 percent of the asset holding distribution, Campbell [1] finds that more than 80 percent of U.S. households hold public equity. However, within the bottom 25 percent of the asset holding distribution less than 40 percent of U.S. households hold public equity. He also finds that participation in equity markets rises substantially with the level of education. Thus, we think that the assumption that the Euler equation for equities holds is better supported for the College education sample that we analyze than for the High School education sample.

\subsection{Stochastic model for earnings}

The statistical model of earnings that we estimate is broadly similar to the model analyzed in Storesletten, Telmer and Yaron [9]. In this model $y_{i, j, t}$ denotes the logarithm of annual earnings for individual $i$ of age $j$ in year $t$. We assume that $y_{i, j, t}$ is comprised of five orthogonal components:

$$
\begin{aligned}
& y_{i, j, t}=\phi_{t}+\kappa_{j}+f_{i}+z_{i, j, t}+\varepsilon_{i, j, t} \\
& z_{i, j, t}=\rho z_{i, j-1, t-1}+\eta_{i, j, t} \\
& z_{i, 0, t}=0 .
\end{aligned}
$$

The first two components are aggregate. $\phi_{t}$ is a stochastic time effect that reflects changes in aggregate labor productivity that are common to all workers. $\kappa_{j}$ is a deterministic experience profile that we model as quartic polynomial in age. 
Table 1

Parameter estimates for idiosyncratic earnings process.

\begin{tabular}{lccc}
\hline & ALL & HS & COL \\
\hline$\sigma_{f}^{2}$ & 0.161 & 0.149 & 0.154 \\
& $(0.011)$ & $(0.015)$ & $(0.013)$ \\
$\rho$ & 0.934 & 0.835 & 0.915 \\
& $(0.025)$ & $(0.067)$ & $(0.030)$ \\
$\sigma_{\eta}^{2}$ & 0.034 & 0.048 & 0.038 \\
& $(0.007)$ & $(0.016)$ & $(0.008)$ \\
$\sigma_{\varepsilon}^{2}$ & 0.128 & 0.125 & 0.111 \\
& $(0.011)$ & $(0.018)$ & $(0.011)$ \\
\hline
\end{tabular}

Notes: Standard errors computed by block bootstrap with 250 repetitions. Reported parameter estimates for $\sigma_{\eta}^{2}$ and $\sigma_{\varepsilon}^{2}$ are averages over 1967 to 1996 . Estimation allows for time effect in both these variances. Standard errors are bootstrap standard errors of the averages. See Appendix B for details.

The remaining three terms are individual-specific. $f_{i} \sim N\left(0, \sigma_{f}^{2}\right)$ is an individual-specific fixed effect, $\eta_{i, j, t} \sim N\left(0, \sigma_{\eta, t}^{2}\right)$ is an idiosyncratic persistent shock and $\varepsilon_{i, j, t} \sim N\left(0, \sigma_{\varepsilon, t}^{2}\right)$ is a purely transitory idiosyncratic shock. ${ }^{4}$

We estimate the model using data on male annual labor earnings from the Panel Study of Income Dynamics (PSID) from 1967 to 1996. We restrict attention to male heads of households between ages 25 and 55. Thus, we value individual earnings over the ages 25 to 55 . Hence in our calculations, human capital values are zero after age 55. We divide the sample into High School (HS) and College (COL) sub-samples, based on their maximum observed completed years of education. Individuals with 12 or fewer years of education are labeled HS while those with more than 12 years are labeled COL. We hence make no distinction between High School dropouts and High School graduates on one hand, and College dropouts, College graduates and postgraduates on the other hand. Our measure of annual gross labor earnings includes pre-tax wages and salaries from all jobs, plus commision, tips, bonuses and overtime, as well as the labor part of income from self-employment. Labor earnings are inflated to 2008 dollars using the CPI All Urban series. Full details can be found in Appendix A.

The model is estimated in two stages. In the first stage we use OLS to estimate the age profile, $\hat{\kappa}_{j}$, and the year effects, $\hat{\phi}_{t}$. Residuals from the first stage are then used to obtain GMM estimates of the remaining parameters, where the moments included are the elements of the autocovariance function for each age/year combination. Full details of the estimation procedure can be found in Appendix B. Parameter estimates are shown in Table 1. These results are consistent with estimates from similar specifications that have been estimated elsewhere in the literature summarized in Meghir and Pistaferri [8].

\footnotetext{
4 When estimating the model we allow for time effects in both the persistent and transitory variance. However, we restrict these to be constant when calculating bounds.
} 
Table 2

Returns summary statistics, 1967 to 2004.

\begin{tabular}{llr}
\hline & Mean & St. dev. \\
\hline $\log \left(1+r_{t}^{f}\right)$ & $1.26 \%$ & $2.40 \%$ \\
$\log \left(1+r_{t}^{e}\right)$ & $5.72 \%$ & $17.50 \%$ \\
\hline
\end{tabular}

Notes: Risk-free rate is average monthly return on 1-month treasury bills, averaged over the calendar year. Equity returns are annual returns on a NYSE/AMEX/NASDAQ value-weighted portfolio including dividends.

\subsection{Asset returns}

We use monthly returns on 1-month treasury bills, averaged over the calendar year, as data for the annual risk-free rate, $r_{t}^{f}$. Equity returns, $r_{t}^{e}$, are annual returns on a value-weighted portfolio of all NYSE, AMEX and NASDAQ stocks including dividends, from 1967-2004. ${ }^{5}$ Real returns on both assets are calculated by adjusting for realized inflation using the same CPI All Urban series that was applied to the earnings data. Table 2 displays summary statistics of the log gross real return for this time period.

\subsection{Joint dynamics of earnings and asset returns}

Although the PSID is an ideal data set for studying the auto-correlation structure of individual earnings, its relatively small sample size and the fact that after 1996 it was converted into a biannual survey means that it is less suited to studying dynamics in the aggregate component $\phi_{t}$ of earnings. Our approach is to retain the PSID as our data source for the idiosyncratic component of earnings but to also analyze an alternative measure of the aggregate component of labor earnings $\phi_{t}$ estimated using Current Population Survey (CPS) data. We estimate this component from CPS data in the same way as we do in the PSID: we run a first stage regression of individual log earnings on a polynomial in age and on time dummy variables. We remove the low-frequency component of our estimate of $\phi_{t}$ by removing a linear trend. ${ }^{6}$ We denote the deviations of the estimate of $\phi_{t}$ from trend as $Y_{t}$. See Appendix A for further details.

We work with demeaned equity returns data of the form $Q_{t}=\log \left(1+r_{t}^{e}\right)$. By construction, our measure of aggregate earnings has mean zero.

The joint dynamics of aggregate earnings and equity returns are assumed to follow an unrestricted first-order vector autoregression (VAR):

$$
\begin{aligned}
& \left(\begin{array}{c}
Y_{t} \\
Q_{t}
\end{array}\right)=A\left(\begin{array}{c}
Y_{t-1} \\
Q_{t-1}
\end{array}\right)+u_{t} \\
& E\left[u_{t} u_{t}^{\prime}\right]=\Sigma .
\end{aligned}
$$

Parameter estimates from this VAR are reported in Table 3 and the implied steady-state dynamics are reported in Table 4. The stochastic properties of the VAR in Table 4 are for the most

\footnotetext{
5 All returns data come from Kenneth French and are available at http://mba.tuck. dartmouth. edu/ pages/faculty/ken. french/data_library.html.

6 We have also examined the sensitivity of our estimates to using a Hodrick-Prescott filter to remove the low-frequency components and obtain very similar results. We choose to focus on the linear trend for the sake of transparency. An alternative approach would have been to work with growth rates rather than levels of the relevant series.
} 
Table 3

Parameter estimates for VAR.

\begin{tabular}{|c|c|c|c|c|c|c|c|}
\hline & & \multicolumn{3}{|l|}{ PSID } & \multicolumn{3}{|l|}{ CPS } \\
\hline & & ALL & HS & $\mathrm{COL}$ & ALL & HS & $\mathrm{COL}$ \\
\hline \multicolumn{8}{|l|}{$A$} \\
\hline & $Y_{t-1}$ & $0.864^{* *}$ & $0.971^{* *}$ & $0.843^{* *}$ & $0.959^{* *}$ & $1.006^{* *}$ & $0.931^{* *}$ \\
\hline & $Q_{t-1}$ & $0.101^{* *}$ & $0.122^{* *}$ & $0.101^{*}$ & $0.076^{* *}$ & $0.081^{* *}$ & $0.078^{* *}$ \\
\hline & $Y_{t-1}$ & $-1.408^{*}$ & $-2.053^{* *}$ & $-1.051^{*}$ & $-1.436^{* *}$ & $-1.389^{* *}$ & $-1.146^{*}$ \\
\hline & $Q_{t-1}$ & -0.193 & -0.244 & -0.165 & -0.107 & -0.099 & -0.078 \\
\hline \multicolumn{8}{|l|}{$\Sigma$} \\
\hline \multicolumn{2}{|c|}{$\overline{\Sigma_{Y Y}\left(\times 10^{-3}\right)}$} & 0.751 & 0.492 & 1.270 & 0.552 & 0.516 & 0.763 \\
\hline \multicolumn{2}{|c|}{$\Sigma_{Q Q}\left(\times 10^{-0}\right)$} & 0.024 & 0.020 & 0.024 & 0.025 & 0.025 & 0.026 \\
\hline \multicolumn{2}{|c|}{$\Sigma_{Y Q}\left(\times 10^{-3}\right)$} & -0.804 & 0.192 & -0.887 & 0.186 & 0.405 & 0.061 \\
\hline
\end{tabular}

Notes: (**) Significant at 1\% level. (*) Significant at 5\% level. PSID data are from 1967-1996, CPS data are from 1967-2004.

Table 4

Implied steady-sate statistics from VAR.

\begin{tabular}{|c|c|c|c|c|c|c|c|}
\hline & & \multicolumn{3}{|l|}{ PSID } & \multicolumn{3}{|l|}{ CPS } \\
\hline & & $\overline{\mathrm{ALL}}$ & HS & $\mathrm{COL}$ & $\overline{\mathrm{ALL}}$ & HS & $\mathrm{COL}$ \\
\hline \multirow{2}{*}{$s d\left(Y_{t}\right)$} & Data & 0.050 & 0.062 & 0.046 & 0.054 & 0.060 & 0.057 \\
\hline & Model & 0.045 & 0.046 & 0.058 & 0.054 & 0.065 & 0.059 \\
\hline \multirow{2}{*}{$s d\left(R_{t}\right):$} & Data & 0.167 & 0.167 & 0.167 & 0.175 & 0.175 & 0.175 \\
\hline & Model & 0.166 & 0.167 & 0.167 & 0.174 & 0.179 & 0.173 \\
\hline \multirow{2}{*}{$\operatorname{corr}\left(Y_{t}, R_{t}\right):$} & Data & -0.364 & -0.325 & -0.394 & -0.370 & -0.344 & -0.343 \\
\hline & Model & -0.390 & -0.427 & -0.359 & -0.359 & -0.411 & -0.323 \\
\hline \multirow{2}{*}{$\operatorname{corr}\left(Y_{t}, Y_{t-1}\right):$} & Data & 0.754 & 0.756 & 0.774 & 0.869 & 0.858 & 0.889 \\
\hline & Model & 0.719 & 0.783 & 0.738 & 0.871 & 0.916 & 0.857 \\
\hline \multirow{2}{*}{$\operatorname{corr}\left(R_{t}, R_{t-1}\right)$} & Data & -0.089 & -0.089 & -0.089 & 0.061 & 0.061 & 0.061 \\
\hline & Model & -0.043 & 0.000 & -0.034 & 0.054 & 0.110 & 0.048 \\
\hline \multirow{2}{*}{$\operatorname{corr}\left(Y_{t}, R_{t-1}\right)$} & Data & -0.030 & -0.065 & 0.019 & -0.117 & -0.102 & -0.105 \\
\hline & Model & 0.033 & 0.023 & -0.012 & -0.100 & -0.192 & -0.072 \\
\hline \multirow{2}{*}{$\operatorname{corr}\left(R_{t}, Y_{t-1}\right)$} & Data & -0.179 & -0.163 & -0.371 & -0.403 & -0.365 & -0.411 \\
\hline & Model & -0.310 & -0.466 & -0.305 & -0.408 & -0.467 & -0.365 \\
\hline
\end{tabular}

Notes: Table shows average moments in the data, together with implied steady-state statistics from the corresponding estimated vector auto-regression. PSID data are from 1967-1996, CPS data are from 1967-2004.

part similar across the two definitions of aggregate earnings. The parameter estimates for all cases reveal a high degree of persistence in aggregate earnings, although the autocorrelation of the aggregate component of earnings in CPS data is slightly higher than that in PSID data (around 0.87 vs 0.75 ). In all specifications we also find a moderate negative contemporaneous correlation (around -0.35 ) between one-period equity returns and aggregate labor income. 


\section{Bounds on values and returns}

\subsection{Volatilty bound on stochastic discount factor}

To compute value bounds we need to set the parameter $b^{2}$ governing the upper bound on the second moment of an agent's stochastic discount factor. A simple rearrangement of the Euler equation produces the well-known restriction below on the coefficient of variation of one period stochastic discount factors. This restriction states that the coefficient of variation is at least as large as the conditional Sharpe ratio, where $R_{j+1}^{x}$ is the excess return on equity

$$
\frac{\sigma_{j}\left(m_{j, j+1}^{*}\right)}{E_{j}\left[m_{j, j+1}^{*}\right]} \geqslant \frac{\left|E_{j}\left[R_{j+1}^{x}\right]\right|}{\sigma_{j}\left(R_{j+1}^{x}\right)} .
$$

We choose values of the parameter $b^{2}$ so that the coefficient of variation of the stochastic discount factor is no larger than a given multiple $(\alpha)$ of the conditional Sharpe ratio. Because our assumed stochastic process for aggregate labor earnings and equity returns features variation in the conditional mean of returns, but not the conditional variance, the conditional Sharpe ratio will vary across realizations of earnings and returns. Since we impose bounds that are constant multiples of the conditional Sharpe ratio, this implies that the raw bound ( $b^{2}$ in the terminology of Section 2) varies across the state space. The set of values for this multiple that we consider is $\alpha \in\{1.1,1.5,2.0,4.0\}$. Thus, we explore a tight upper bound that allows only slightly more variation than is needed to satisfy the Euler equations for equity and debt $(\alpha=1.1)$ as well as those that allow substantially more variation $(\alpha=4)$. A tight upper bound means that there is only a little room for the stochastic discount factor to move with or against the orthogonal component of earnings.

\subsection{Properties of value bounds}

To calculate bounds on human capital values, we use recursive methods to solve for the upper and lower bounds at each age at each point in the state space for earnings and returns. ${ }^{7} \mathrm{We}$ take as given that earnings and returns follow the statistical model estimated in Section 4 . We then simulate a large number of realizations of the idiosyncratic earnings shocks, attaching to each simulated agent his own simulated path for the aggregate component of earnings and the return on the risky asset. We report the mean of the upper and lower bounds at each age. All bounds are reported with a value for the individual-specific fixed effect equal to zero (i.e. $f_{i}=0$ ). Bounds for other values for the fixed effect can be obtained by scaling the upper and lower bounds proportionately using $\exp \left(f_{i}\right)$. All of the results we report are based on the CPS estimates from Table 3 for the aggregates (returns and aggregate earnings) and Table 1 estimates for the idiosyncratic component of earnings.

Fig. 2 reports the upper and lower bounds for the High School and College subsamples and for the combined sample. Along with the bounds, we plot the expected discounted value for earnings. This is a natural benchmark with which to compare the size of the bounds. It reflects the value that one would attach to an individual's labor earnings if they were discounted at the risk-free rate.

\footnotetext{
7 See Appendix C for computational details.
} 


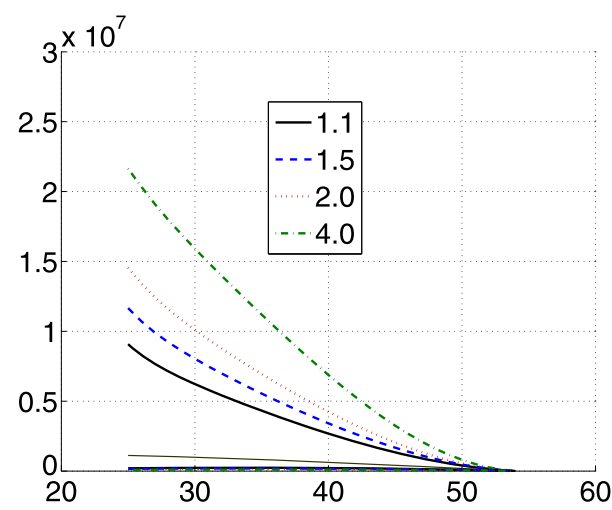

(a) High School

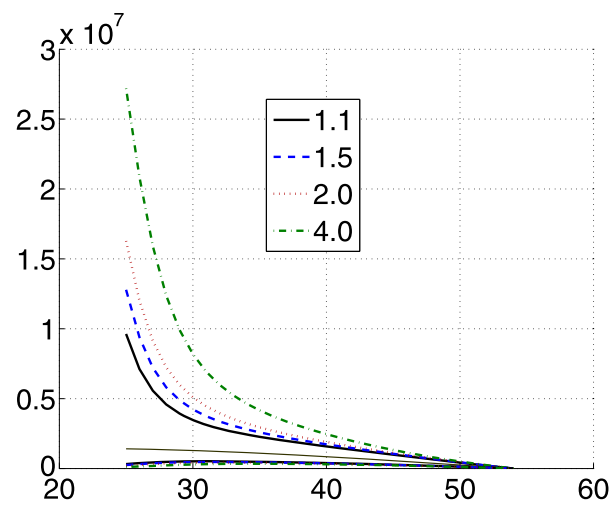

(c) Full Sample

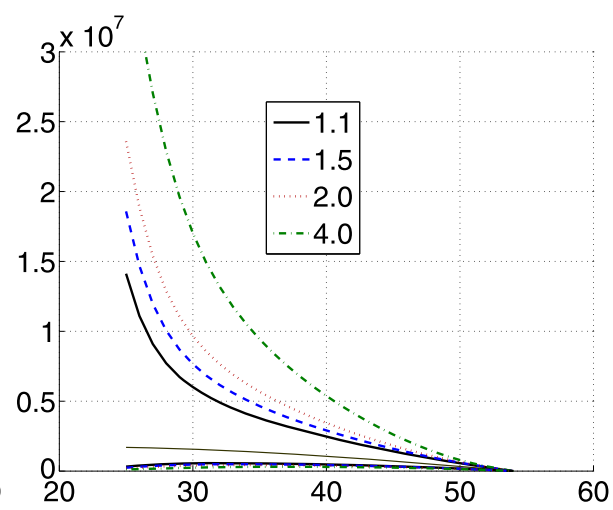

(b) College

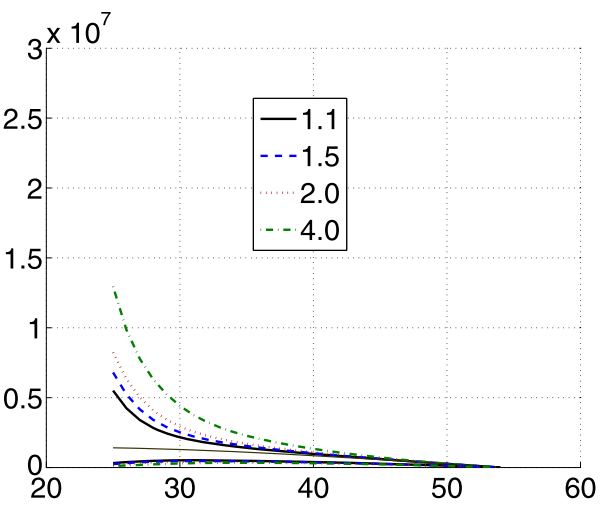

(d) Full Sample (with additional constraint)

Notes: All plots show upper and lower bounds for the value of human capital as a function of age. Units are 2008 dollars. Bounds are reported for multiples of the conditional Sharpe ratio in the set $\{1.1,1.5,2,4\}$. The thin black line is the expected present value of earnings discounted at the risk-free rate. See text for further details.

Fig. 2. Bounds on human capital values. (For interpretation of the references to color in this figure legend, the reader is referred to the web version of this article.)

We find that value bounds are wide. Even with our most restrictive bound on the volatility of the stochastic discount factor, the gap between the upper and lower value bound at young ages is far larger than the difference in the expected discounted value of earnings across education groups. This implies that the restrictions, highlighted in Section 2, coming from asset returns and earnings alone only loosely pin down the value of human capital. This holds both in the full sample and for both education groups.

Note, however, that the width of the value bounds, and their dependence on the level of $\alpha$, stem strongly from the upper bound. Large values for the upper bound are attained by stochastic discount factors that have a strong positive covariation with earnings. In Section 3.4 we discuss that in certain cases theory allows one to impose additional restrictions that rule out positive covariation of various types. We now impose that the component of earnings which is orthogonal to asset returns does not covary positively with the stochastic discount factor. This is a stronger condition than was formally justified. Recall that Claim 2 from Section 3 ruled out positive covariation for only the purely temporary component of earnings variation. 
Table 5

Bounds on expected lifetime returns (\%).

\begin{tabular}{|c|c|c|c|c|c|c|c|}
\hline \multirow{2}{*}{$\begin{array}{l}\text { Bound } \\
(\alpha)\end{array}$} & \multicolumn{2}{|c|}{ High School } & \multicolumn{2}{|c|}{ College } & \multicolumn{3}{|c|}{ Full sample } \\
\hline & Lower & Upper & Lower & Upper & Lower & Lower* & Upper \\
\hline 1.1 & -12.4 & 23.6 & -11.2 & 17.5 & -10.3 & -5.1 & 17.0 \\
\hline 1.5 & -13.7 & 29.7 & -12.5 & 20.6 & -11.6 & -6.1 & 19.4 \\
\hline 2.0 & -14.8 & 36.1 & -13.7 & 23.7 & -12.7 & -6.9 & 21.8 \\
\hline 4.0 & -16.8 & 55.5 & -15.9 & 32.0 & -14.9 & -9.1 & 27.3 \\
\hline
\end{tabular}

Notes: "Lower*" refers to bounds calculated by imposing the additional restriction that the covariance between the orthogonal component of earnings and the stochastic discount factor is non-positive.

Fig. 2(d) shows that imposing this additional constraint substantially tightens the upper bound for values. For example, the average reduction in the upper bound for the full sample with $\alpha=2.0$ is a factor of 1.6. The additional constraint on the set of stochastic discount factors does not alter any of the lower bounds.

\subsection{Properties of return bounds}

We construct upper and lower bounds on returns. Returns bounds are calculated by simulating a large number of paths for the upper and lower bounds on values, and calculating the bounds on returns that are implied by each of these paths based on Theorem 1 . We report the means of these lifetime return bounds in Table 5 and note that (i) they are wide and (ii) in no case is the lower bound positive. These findings further stress the results from the bounds on values: even with tight restrictions on the volatility of the stochastic discount factor, equity returns and the risk-free rate do not imply tight bounds for the return on human capital.

It is also possible to construct a life-cycle profile for return bounds. After any sequence of shock realizations up to age $j$, one can define a lifetime return after that age and history as the geometric average of future returns to human capital. We construct bounds for these returns and plot the mean return bound at each age in Fig. 3. The bounds tend to fan out with age. Thus, examining returns over a shorter interval of the remaining lifetime does not result in tighter bounds than those in Table 5. This holds for the full sample and for both education levels. We note that the lower bound on the return at the end of the working lifetime (age 55) in Fig. 3(d) is positive. This is consistent with the general spirit of Claim 1 from Section 3. Claim 1 says that the orthogonal component of earnings in the last period, given returns, cannot covary positively with the agent's discount factor and thus this component of earnings can only serve to lower the value of an individual's human capital in the second to last period of the working lifetime.

\section{What type of earnings risk matters for bounds?}

In this section we assess which factors are important for the width of the bounds reported in Section 5. We first show that idiosyncratic risk, rather than aggregate risk, accounts for almost all of the width of the bounds. We then argue that early in life, persistent shocks account for most of the gap between the upper and lower bound, while closer to retirement, transitory shocks are more important.

Fig. 4 displays value bounds for the combined sample from a version of the model without aggregate earnings shocks (4(a)) and without idiosyncratic shocks (4(b)). To calculate bounds in the absence of aggregate shocks, we construct an $\mathrm{AR}(1)$ process for equity returns that has 


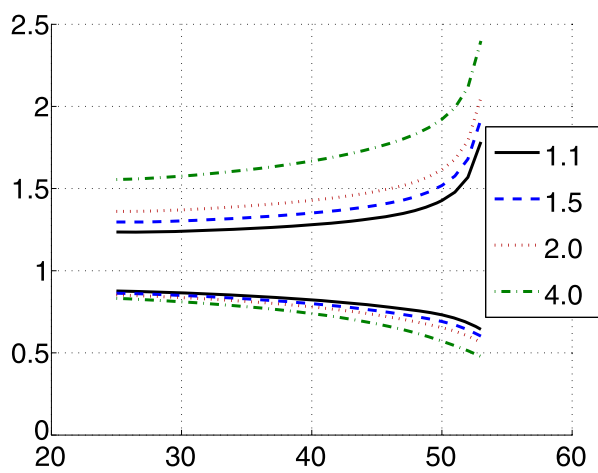

(a) High School

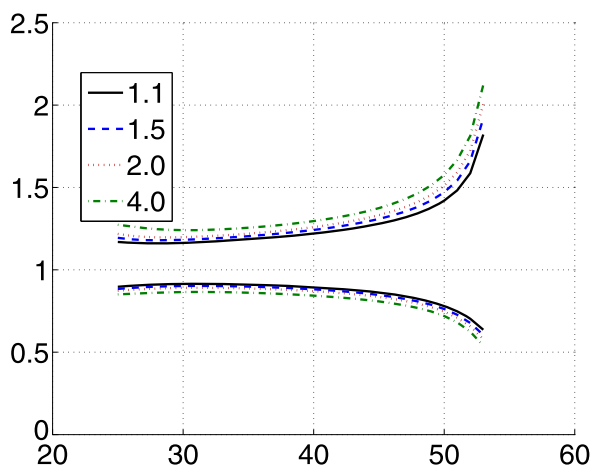

(c) Full Sample

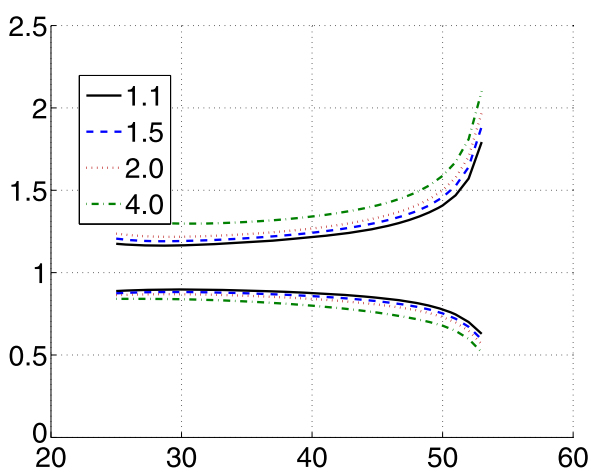

(b) College

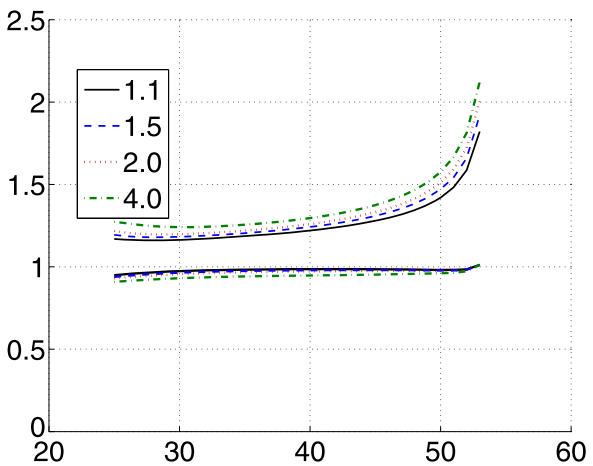

(d) Full Sample (with additional constraint)

Notes: All plots show upper and lower bounds for the gross expected lifetime return to human capital as a function of age. Bounds are reported for multiples of the conditional Sharpe ratio in the set $\{1.1,1.5,2,4\}$. See text for further details.

Fig. 3. Bounds on human capital returns.

unconditional moments that are the same as the marginal moments for equity returns from the estimated VAR. Removing aggregate shocks narrows the bounds by roughly a factor of 2, but the bounds are still wide.

In contrast, when the only source of earnings risk is aggregate shocks (i.e. idiosyncratic risk is completely removed) the value of human capital is almost uniquely determined. Bounds are not degenerate: they are just so narrow that when plotted on the same scale as the original bounds they appear to be a single line. The value bounds for this case imply that lifetime expected net returns must lie between $0.25 \%$ and $2.50 \% .^{8}$

We conclude that with an empirically plausible joint stochastic process for aggregate earnings and equity returns, it is precisely idiosyncratic risk that makes it difficult to pin down the value and return to human capital using data on asset returns and earnings alone. Absent idiosyncratic risk, the weak restrictions we impose on stochastic discount factors, that are used in finance, would usefully pin down human capital values and returns.

\footnotetext{
8 Repeating this exercise, we find lifetime expected return bounds of $(0.10,2.6)$ for the College sample and $(0.23,2.6)$ for the High School sample.
} 


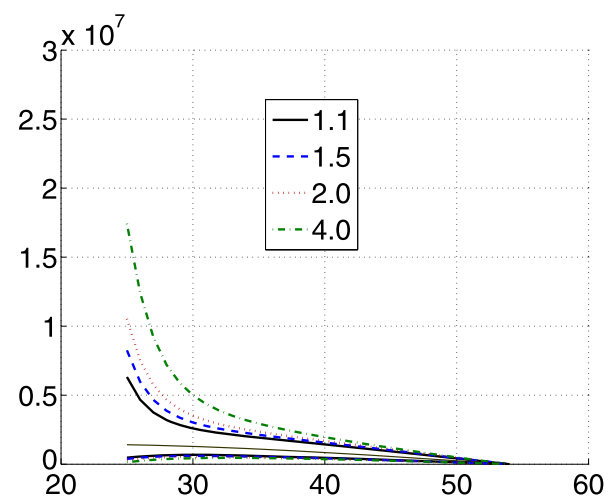

(a) Only Idiosyncratic Shocks: Values

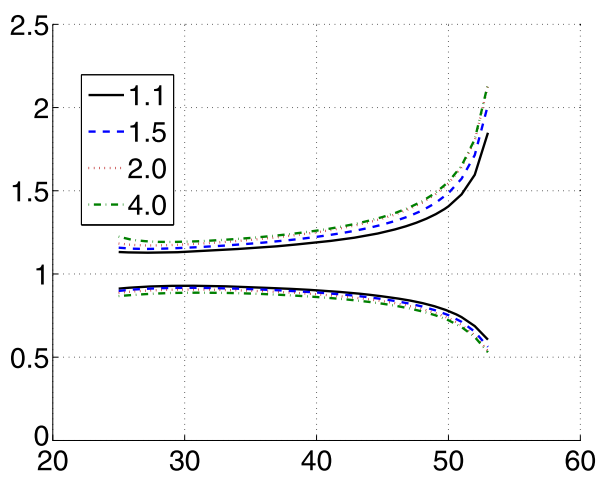

(c) Only Idiosyncratic Shocks: Returns

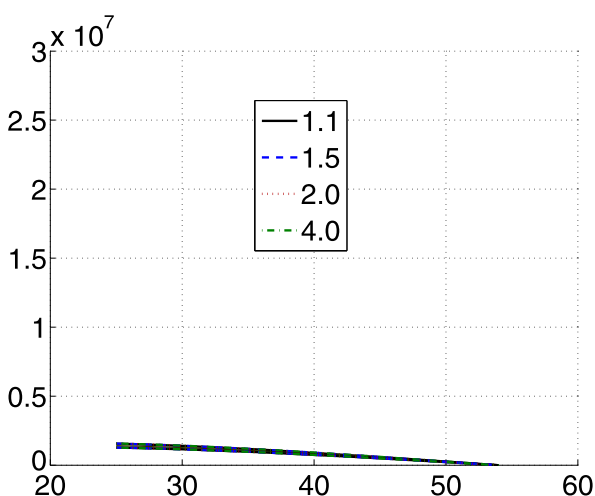

(b) Only Aggregate Shocks: Values

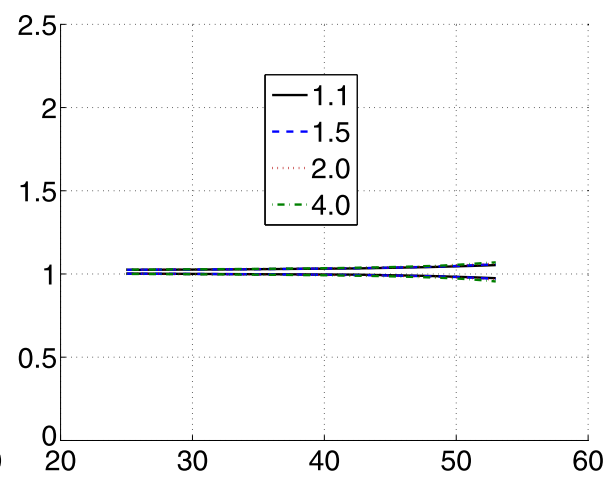

(d) Only Aggregate Shocks: Returns

Notes: Plots (a) and (b) show upper and lower bounds for the value of human capital as a function of age. Units are 2008 dollars. Plots (c) and (d) show upper and lower bounds for the gross expected lifetime return to human capital as a function of age. Bounds are reported for multiples of the conditional Sharpe ratio in the set $\{1.1,1.5,2,4\}$. The thin black line is the expected present value of earnings discounted at the risk-free rate. See text for further details.

Fig. 4. Human capital values and returns: Aggregate vs idiosyncratic shocks. (For interpretation of the references to color in this figure legend, the reader is referred to the web version of this article.)

Next, we investigate which type of idiosyncratic earnings risk is responsible for the width of bounds. Fig. 5 displays value bounds when we retain only persistent earnings shocks (5(a)) and only transitory earnings shocks $(5(b))$. Overall, both types of risk appear to contribute to wide bounds. However, there are important differences over the life cycle. For young agents, there are many remaining years to feel the effects of persistent shocks, so these contribute a great deal to the width of bounds. For older agents, persistent shocks are less important, because there are fewer years to realize their effects.

\section{Final remarks}

We have constructed bounds on the value of human capital and then used these bounds to construct bounds on the lifetime return to human capital. The bounds are derived from knowledge of the set of traded assets, the joint stochastic process for individual earnings and asset returns, as well as three assumptions about an individual's stochastic discount factors: they are (i) non- 


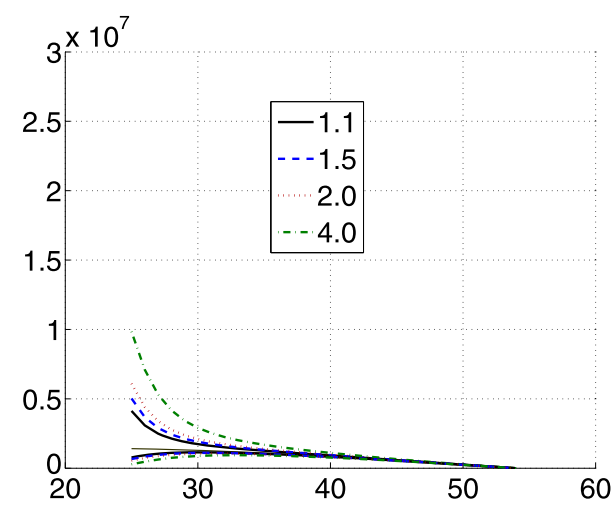

(a) Only Persistent Shocks

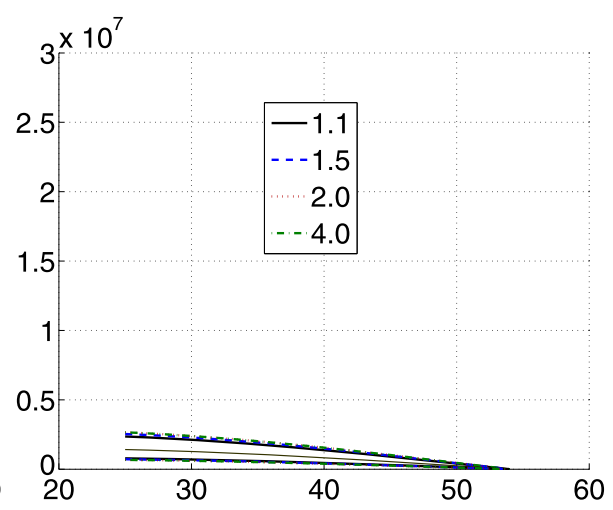

(b) Only Transitory Shocks

Notes: Plots show upper and lower bounds for the value of human capital as a function of age. Units are 2008 dollars. Bounds are reported for multiples of the conditional Sharpe ratio in the set $\{1.1,1.5,2,4\}$. The thin black line is the expected present value of earnings discounted at the risk-free rate. See text for further details.

Fig. 5. Persistent vs transitory idiosyncratic risk. (For interpretation of the references to color in this figure legend, the reader is referred to the web version of this article.)

negative, (ii) satisfy an Euler equation for each asset and (iii) have a second moment no larger than some pre-specified upper bound.

Using U.S. data, we find that value and return bounds are quite wide. Even allowing for only slightly more variation in the stochastic discount factor than is needed to price equity and debt, we find that earnings and asset returns data can only restrict the mean lifetime return on human capital to lie between -5 percent and 17 percent per year. The vast majority of the gap is due to the idiosyncratic component of earnings risk. Absent the idiosyncratic component of earnings risk, the average lifetime return on human capital is between 0.25 and 2.5 percent per year - not far from the average risk-free rate. One of the main messages of these findings is that to learn something sharper about the return to an individual's human capital will require a structural approach. Huggett and Kaplan [6] take up this challenge and use a fully specified structural model with idiosyncratic and aggregate sources of earnings risk to measure the value and return to human capital.

We highlight two challenges to the empirical findings of this paper that might be taken up in future work. First, the statistical model of earnings analyzed in Section 4 may overstate the magnitude of persistent idiosyncratic earnings shocks. Huggett, Ventura and Yaron [7] argue that learning ability differences across individuals can account for much of the large rise in the variance of log earnings observed over the working lifetime. Thus, the role of persistent shocks may be substantially smaller than what we infer in Table 1 . Second, we have assumed that the aggregate component of male earnings has a deterministic trend. Future work can investigate the possibility of stochastic trends or cointegration between the aggregate component of earnings and equity returns. These possibilities may give the aggregate component of earnings a larger role in producing higher mean returns to human capital.

\section{Appendix A. Data and sample selection}

We use the core PSID sample from waves 1968 to 1997, which refer to earnings in years 1967 to 1996. After 1997 the PSID became a bi-annual survey, hence we exclude the more recent waves. We restrict attention to male heads of household between the ages of 25 and 55 with 
annual labor income of at least $\$ 1000$ in 2008 dollars. Our measure of annual labor income includes pre-tax wages and salaries from all jobs, plus commission, tips, bonuses and overtime, as well as the labor part of income from self-employment. Our final sample contains 54,589 observations on 5,130 individuals. The median number of annual observations per individual is 9. We construct three education samples: one comprising all males (ALL), one comprising males with 12 or fewer years of education (HS) and one comprising males with more than 12 years of education (COL). Our CPS data comes from the IPUMS database of March Outgoing Rotation Groups. We use data on earnings from 1967 to 2004. The aggregate components of labor earnings for each subsample are measured as the coefficients on year dummies in a regression that is analogous to the one described below.

\section{Appendix B. Estimation of idiosyncratic earnings model}

Estimation is done in two stages. In the first stage we estimate $\phi_{t}$ and $\kappa_{j}$ by regressing $\log$ real annual earnings on a quartic polynomial in age and a full set of year dummies. This is done separately for the three education samples. Residuals from the first-stage regression are then used to estimate the remaining parameters of the individual earnings equation, $\left(\rho, \sigma_{f}^{2},\left\{\sigma_{\eta, t}^{2}\right\},\left\{\sigma_{\varepsilon, t}^{2}\right\}\right)$. The auto-covariance function for residual log-earnings is calculated for up to 10 lags for every age/year combination. For this purpose, individuals are grouped into 5-year age cells so that when calculating covariances at age $j$, individuals aged $j \in[j-2, j+2]$ are used. Only cells with at least 30 observations are retained. A GMM estimator is then used to estimate the parameters, where the moments included are the elements of the auto-covariance function. The moments are weighted by $n_{j, t, l}^{0.5}$ where $n_{j, t, l}$ is the number of observations used to calculate the covariance at lag $l$ in year $t$ for age $j$. Since the permanent shock and the transitory shock are not separately identified in the last period, we assume that $\sigma_{\eta, T}^{2}=\sigma_{\eta, T-1}^{2}$. Individuals aged 25 to 55 are used to construct the empirical auto-covariance functions. This means that variances and covariance from ages 27 to 53 are effectively used in the estimation. Standard errors are calculated by bootstrap with 250 repetitions, thus accounting for estimation error induced by the first-stage estimation.

\section{Appendix C. Computational details}

Bounds are calculated using backwards recursion, starting from a value of zero in retirement. We discretize the state space using 5 points for the return on the risky asset, 5 points for the aggregate component of earnings, 5 points for the persistent idiosyncratic component and 5 points for the transitory component. Bounds are calculated for a value of the fixed effect equal to zero. Note that bounds for other values of the fixed effect are just multiples of these bounds. With a discrete state space for earnings and asset returns the calculation of bounds can be recast as a linear optimization problem in the state-specific values of the stochastic discount factor, subject to linear constraints (the Euler equations), non-negativity constraints and a single quadratic constraint (the volatility bound on the SDF). We use a semi-definite programming algorithm (SeDuMi 1.21 implemented in Matlab) to perform this optimization.

Specifically, we solve the following finite horizon dynamic programming problem, where $\tilde{x}_{j}$ is the vector of state variables that is relevant for forecasting future earnings and asset returns, $\varepsilon_{j}$ is the transitory component of earnings and $x_{j}=\left(\tilde{x}_{j}, \varepsilon_{j}\right)$. Note that $\tilde{x}_{j}$ has three components: two from the VAR and one from the persistent idiosyncratic shock. We use $m_{j}$ to denote oneperiod stochastic discount factors, $e_{j}$ to denote earnings, $R_{j}^{e}$ to denote the return on equities and $R^{f}$ to denote the risk-free rate. To calculate upper bounds on values we solve 


$$
v_{j}^{u}\left(\tilde{x}_{j}\right)=\max _{\left\{m_{j+1}\left(x_{j+1}\right)\right\}} \sum_{x_{j+1}} m_{j+1}\left(x_{j+1}\right)\left[e_{j+1}\left(x_{j+1}\right)+v_{j+1}^{u}\left(\tilde{x}_{j+1}\right)\right] \operatorname{Pr}\left(x_{j+1} \mid \tilde{x}_{j}\right)
$$

subject to:

$$
\begin{aligned}
& m_{j+1}\left(x_{j+1}\right) \geqslant 0 \quad \forall x_{j+1} \\
& \sum_{x_{j+1}} m_{j+1}\left(x_{j+1}\right) R_{j+1}^{e}\left(x_{j+1}\right) \operatorname{Pr}\left(x_{j+1} \mid \tilde{x}_{j}\right)=1 \\
& R_{j+1}^{f} \sum_{x_{j+1}} m_{j+1}\left(x_{j+1}\right) \operatorname{Pr}\left(x_{j+1} \mid \tilde{x}_{j}\right)=1 \\
& \sum_{x_{j+1}} m_{j+1}\left(x_{j+1}\right)^{2} \operatorname{Pr}\left(x_{j+1} \mid \tilde{x}_{j}\right) \leqslant b^{2} .
\end{aligned}
$$

Lower bounds are calculated by replacing the max operator with a min operator. We impose the constraint that the covariance between the stochastic discount factor and the orthogonal component of earnings is non-positive by adding the following constraint

$$
\sum_{x_{j+1}} m_{j+1}\left(x_{j+1}\right) \tilde{e}_{j+1}\left(x_{j+1}\right) \operatorname{Pr}\left(x_{j+1} \mid \tilde{x}_{j}\right) \leqslant \frac{1}{R^{f}} \sum_{x_{j+1}} \tilde{e}_{j+1}\left(x_{j+1}\right) \operatorname{Pr}\left(x_{j+1} \mid \tilde{x}_{j}\right)
$$

where $\tilde{e}_{j}$ is the residual from a projection of $e_{j}$ on $\left(R^{f}, R_{j}^{e}\right)$.

\section{Appendix D. Proof of Theorem 1}

(i) We focus on proving the upper bound result as the lower bound result follows by a parallel argument. The inequality below provides an upper bound to $R_{\text {life }}(m), \forall m \in M$, given $\bar{\omega} \in \Omega$. In stating the inequality, we note that $\left(R_{\text {life }}(m), v_{j}(m), v_{j}^{l}, e_{j}\right)$ are all random variables. We hide this dependence on the realization $\bar{\omega} \in \Omega$ for notational simplicity

$$
R_{\text {life }}(m)=\left[\frac{e_{J}}{v_{1}(m)} \prod_{j=2}^{J-1}\left(1+\frac{e_{j}}{v_{j}(m)}\right)\right]^{\frac{1}{J-1}} \leqslant\left[\frac{e_{J}}{v_{1}^{l}} \prod_{j=2}^{J-1}\left(1+\frac{e_{j}}{v_{j}^{l}}\right)\right]^{\frac{1}{J-1}} .
$$

This candidate upper bound is the least upper bound if $\forall \epsilon>0, \exists m \in M$ such that condition $v_{j}(m)-v_{j}^{l}<\epsilon, \forall j$ holds, given $\bar{\omega} \in \Omega$. This follows as $\left[\frac{e_{J}}{v_{1}(m)} \prod_{j=2}^{J-1}\left(1+\frac{e_{j}}{v_{j}(m)}\right)\right]^{\frac{1}{J-1}}$ is continuous in these strictly positive realized values.

We now construct an $m \in M$ with this property. Given any $\epsilon>0$, suppose we have an $m^{1} \in M$ such that $v_{j}\left(m^{1}\right)-v_{j}^{l}<\epsilon$ for all $j \leqslant k$, given $\bar{\omega} \in \Omega$. This clearly holds for $k=1$. By the definition of the infimum, $\exists m^{2} \in M$ such that $v_{k+1}\left(m^{2}\right)-v_{k+1}^{l}<\epsilon$, given $\bar{\omega} \in \Omega$. Define $m^{3}$, as indicated below, so that $v_{j}\left(m^{3}\right)-v_{j}^{l}<\epsilon$ for all $j \leqslant k+1$. Clearly, $m^{3} \in M$ as it is constructed from $m^{1}, m^{2} \in M$. Repeating this construction to apply for all ages, establishes that the relevant property holds:

$$
\begin{aligned}
& m_{j, j+1}^{3}(\omega)=\left\{\begin{array}{l}
m_{j, j+1}^{2}(\omega) \quad \forall j \geqslant k+1, \forall \omega \in F_{k+1} \text { if } v_{k+1}\left(m^{2}\right)(\bar{\omega})<v_{k+1}\left(m^{1}\right)(\bar{\omega}) \\
m_{j, j+1}^{1}(\omega) \quad \text { all other cases }
\end{array}\right. \\
& F_{k} \equiv\left\{\omega \in \Omega: \forall j \leqslant k, e_{j}(\omega)=e_{j}(\bar{\omega}) \text { and } R_{j}^{i}(\omega)=R_{j}^{i}(\bar{\omega}), \forall i \in I\right\} .
\end{aligned}
$$

(ii) Since $m^{*} \in M, R_{\text {life }}\left(m^{*}\right) \in\left[R_{\text {life }}^{l}, R_{\text {life }}^{u}\right]$. 


\section{References}

[1] J. Campbell, Household finance, J. Finance 61 (4) (2006) 1553-1604.

[2] J. Cochrane, Asset Pricing, Princeton University Press, 2001.

[3] J. Cochrane, J. Saa-Requejo, Beyond arbitrage: Good-deal asset price bounds in incomplete markets, J. Polit. Economy 108 (1) (2000) 79-119.

[4] G. Constantinides, D. Duffie, Asset pricing with heterogeneous consumers, J. Polit. Economy 104 (2) (1996) 219 240.

[5] L. Hansen, R. Jagannathan, Implications of security market data for models of dynamic economies, J. Polit. Economy 99 (2) (1991) 225-262.

[6] M. Huggett, G. Kaplan, The money value of a man, Manuscript, Georgetown University, 2010.

[7] M. Huggett, G. Ventura, A. Yaron, Sources of lifetime inequality, NBER Working Paper, 2007.

[8] C. Meghir, L. Pistaferri, Earnings, Consumption and Lifecycle Choices, NBER Working Papers, 2010.

[9] K. Storesletten, C. Telmer, A. Yaron, Consumption and risk sharing over the life cycle, J. Monet. Econ. 51 (3) (2004) 609-633. 